\title{
Reduction Mechanism of Sulfur in Lithium-Sulfur Battery: from Elemental Sulfur to Polysulfide
}

Dong Zheng ${ }^{\mathrm{a}}$, Xuran Zhang ${ }^{\mathrm{b}}$, Jiankun Wang ${ }^{\mathrm{c}}$, Deyu Qu ${ }^{\mathrm{b}, *}$, Xiaoqing Yang ${ }^{\mathrm{d}}$, Deyang $\mathrm{Qu}^{\mathrm{a}, *}$

${ }^{a}$ Department of Mechanical Engineering, College of Engineering and Applied Science, University of Wisconsin Milwaukee, Milwaukee, WI 53211, USA.

${ }^{\mathrm{b}}$ Department of Chemistry, School of Chemistry, Chemical Engineering and Life Science, Wuhan University of Technology, Wuhan 430070, Hubei, P.R. China

${ }^{\mathrm{c}}$ Department of Applied Chemistry, School of Advanced Materials and Nanotechnology, Xidian University, Xi'an 710126, Shanxi, P.R. China

${ }^{d}$ Chemistry Department, Brookhaven National Laboratory, Upton, NY 11973, USA

*Corresponding Author: deyuquwuhan@163.com; qud@uwm.edu

\begin{abstract}
:
The polysulfide ions formed during the first reduction wave of sulfur in Li-S battery were determined through both in-situ and ex-situ derivatization of polysulfides. By comparing the cyclic voltammetric results with and without the derivatization reagent (methyl triflate) as well as the in-situ and ex-situ derivatization results under potentiostatic condition, in-situ derivatization was found to be more appropriate than its ex-situ counterpart, since subsequent fast chemical reactions between the polysulfides and sulfur may occur during the timeframe of ex-situ procedures. It was found that the major polysulfide ions formed at the first reduction wave of elemental sulfur were the $\mathrm{S}_{4}{ }^{2-}$ and $\mathrm{S}_{5}{ }^{2-}$ species, while the widely accepted reduction products of $\mathrm{S}_{8}{ }^{2-}$ and $\mathrm{S}_{6}{ }^{2-}$ for the first reduction wave were in low abundance.
\end{abstract}

Keywords: reduction of Sulfur, Lithium-Sulfur battery, polysulfide, HPLC 


\section{Introduction}

Recently, rechargeable lithium sulfur (Li-S) and lithium air (Li-Air) batteries have drawn significant attention due to their high theoretical energy density [1]. Both batteries are considered to be potential candidates to replace state-of-art Li-ion batteries in electric vehicles (EVs). Although oxygen and sulfur are in the same group on the periodic table, the electrochemical redox reactions of these two are quite different. It's well known that the redox reaction of sulfur is one of the most complicated redox reactions and its mechanism is still not fully understood. It's believed that the reduction of the most stable form of elemental sulfur, cyclooctasulfur $\left(\mathrm{S}_{8 c}\right)$, is a multistep reduction. The cyclooctasulfur is first electrochemically reduced into long chain linear polysulfides by the cleavage of the sulfur ring. The long chain sulfides are then further reduced into shorter chain polysulfides at a different potential [2-12]. Correspondingly there are two reduction waves observed in the cyclic voltammetry and two discharge plateaus observed between 1.5 to $3.0 \mathrm{~V} \mathrm{vs.} \mathrm{Li} / \mathrm{Li}^{+}$in the discharge profile of a Li-S battery. We demonstrate in this paper that the stepwise sulfur reduction mechanism may not be accurate.

Unlike Li-ion cathode materials which are based on Li ion insertion, the redox reaction of sulfur involves polysulfides dissolution and re-deposition. Therefore, the sulfur cathode experiences compositional, structural, and surface alteration during charge and discharge. Due to the lack of a reliable analytical method for the quantitative and qualitative determination of the soluble polysulfides formed during the various stages of cell operation, the mechanism for the sulfur redox reaction is still debatable; for example, whether the very first charge transfer reaction is the electrochemical reduction of $\mathrm{S}_{8 \mathrm{c}}$ yielding linear $\mathrm{S}_{8}{ }^{2-}$ [8]. To fully understand the stepwise reduction of elemental sulfur in the Li-S battery, substantial fundamental research has been done by means of electrochemistry [4,6,13-15], UV-Vis spectroscopy [2,3,5,7-9,12,16,17], Raman [10,11,18], ESR [19], XRD [10,19-22], XAS 
$[19,22]$, as well as the theoretical calculations [23-25]. These studies revealed the complexity associated with the reduction of sulfur. Different mechanisms of reduction were proposed although most of them lacked direct and clear experimental proof. There are three mainstream mechanisms proposed to explain the first reduction wave at around $2.3 \mathrm{~V}$ vs. Li as shown in schemes 1 to $3: 1$ ) 2-electron electrochemical reduction process followed by chemical reactions (abbreviated 2EC) [5,6,9,13,16,18]; 2) 1-electron electrochemical reduction process followed first by a chemical reaction and then by another one-electron electrochemical reduction (abbreviated ECE) [7,17]; and 3) two successive 1-electron electrochemical reduction processes followed by a chemical reaction (abbreviated EEC) [4,8,14,15]. Although similar analytical and electrochemical methods were used, different observations were reported, thus the different mechanisms described above were proposed according to the corresponding results. It is worth to emphasizing that the existence of $\mathrm{S}_{8}{ }^{-}{ }^{-}$radicals have never been proven experimentally, although the radicals with shorter sulfur chain length e.g. $\mathrm{S}_{3}{ }^{-}$ were detected by ESR experiments [19].

\section{[Schemes 1 to 3 ]}

In the investigation of polysulfide species in an aqueous system, Lev et al reported a derivatization method tandem with separation and identification by HPLC [26-29]. Through reaction with methyl triflate, or methyl iodide, the polysulfide anions were derivatized into different dimethyl polysulfides. Based on the sulfur chain length in the dimethyl polysulfides, the baseline separation of different dimethyl polysulfides can be achieved by HPLC and each dimethyl polysulfide in the chromatogram can be identified based on the relationship between retention time and sulfur chain length. Both Barchasz et al [30] and our group [31] recently reported the investigation of the discharge mechanism of the Li-S battery using ex-situ derivatization coupled with HPLC-MS. All eight polysulfide ions and elemental sulfur can be separated and identified by HPLC-MS after derivatization. 
In this work, for the first time in-situ derivatization with HPLC was used to investigate the mechanism of sulfur reduction. By comparing the in-situ and ex-situ derivatization results under potentiostatic condition, the electrochemical mechanism of the first reduction wave of sulfur was discussed.

\section{Experimental}

\subsection{Chemicals.}

Sulfur(from Fisher Scientific), lithium metal, lithium sulfide $\left(\mathrm{Li}_{2} \mathrm{~S}\right)$, HPLC grade methanol, HPLC grade water, methyl triflate, anhydrous Dimethoxyethane (DME) (from Sigma Aldrich), and lithium bis(trifluoromethane) sulfonimide (LiTFSi, battery grade from FERRO) were purchased and used without further treatment.

\subsection{Sample preparation and methods.}

Three catholyte solutions were prepared. Catholyte A and B were made by mixing excess amount of $\mathrm{Li}_{2} \mathrm{~S}$ with different amounts of elemental sulfur $(\mathrm{A}=0.0194 \mathrm{~g}, \mathrm{~B}=0.0973 \mathrm{~g})$ in 20ml 1M LiTFSi/DME. Catholyte C was a sulfur saturated LiTFSi/DME solution.

Cyclic voltammetry (CV) was carried out using an AutoLab PGSTAT30 and recorded by the Nova software (version 1.7 from Metrohm). The electrochemical cell, with threeelectrode configuration, was assembled and operated under Ar. The working electrode was a glassy carbon disk electrode, the reference and counter electrodes were lithium metal. The potential range for the $\mathrm{CV}$ was $1.5 \mathrm{~V}$ to $3.3 \mathrm{~V}\left(\mathrm{vs} \mathrm{Li} / \mathrm{Li}^{+}\right.$) and $\mathrm{DME}$ was used as the solvent.

The discharge of the Li-S battery was performed with an 8-channel Arbin battery tester. The cylindrical Li-S batteries consisted of three electrodes: the cathode was carbon felt with a geometric area of $3.14 \mathrm{~cm}^{2}$ (BET surface area is about $111.4 \mathrm{~cm}^{2}$ ), the anode and the reference electrode were lithium metal. The total volume for electrolyte in the Li-S battery was $10 \mathrm{ml}$. Catholyte $\mathrm{C}$ was used as the electrolyte and the source of sulfur. For the in-situ 
derivatization, methyl triflate was added into the electrolyte with sulfur and the final concentration of methyl triflate was about $20 \mathrm{mM}$. The Li-S battery was then discharged under potentiostatic conditions (2.3V vs $\left.\mathrm{Li} / \mathrm{Li}^{+}\right)$. For ex-situ derivatization, Li-S battery without methyl triflate in the sulfur saturated electrolyte was discharged under potentiostatic condition (2.3V vs $\mathrm{Li} / \mathrm{Li}^{+}$). After the discharge, the methyl triflate-added electrolyte (in-situ derivatization) was directly analyzed by HPLC UV and MS without any further treatment; and the electrolytes without methyl triflate (ex-situ derivatization) were derivatized by methyl triflate before analysis by HPLC UV and MS. The Li-S batteries were assembled and tested in an Ar-filled glovebox, as was the ex-situ derivatization.

Agilent 1200 quaternary pump with Agilent G1329A autosampler was used to deliver methanol/water mobile phase through a Waters Symmetry $\mathrm{C}_{18}$ column (from Waters, $\mathrm{C}_{18}$, 4.6*50mm, 5um) at flow rate of $0.70 \mathrm{~mL} / \mathrm{min}$. The injection volume was $4 \mathrm{uL}$. A binary gradient mobile phase was used to elute the injected sample out under the following conditions: at 0 min 25\% methanol (75\% water); at 10 min 100\% methanol; at 20 min 100\% methanol; at 21 min 25\% methanol. All flow from the HPLC was first introduced through an Agilent 1260 Diode Array Detector (DAD) and then introduced into the Agilent 6130 quadrupole mass spectrometer (MS) with an Atmospheric Pressure Chemical Ionization (APCI) source. For the DAD detector, the chromatograms of five sets of different wavelength were recorded at: $210 \mathrm{~nm}, 230 \mathrm{~nm}, 254 \mathrm{~nm}, 280 \mathrm{~nm}$, and $300 \mathrm{~nm}$. Only results from the $254 \mathrm{~nm}$ recording were shown and discussed in this work, since all other results at other wavelengths show similar chromatograms (shown in Figure S-3 in the supplemental information). For the MS detector, APCI negative mode was set as follow: Capillary Voltage 3kV, Corona Current $15 \mathrm{uA}$, Drying gas temperature $250^{\circ} \mathrm{C}$, Vaporizer temperature $250^{\circ} \mathrm{C}$, nitrogen gas flow was 12L/min for Drying gas flow, Nebulizer pressure was $35 \mathrm{psi}$. The $\mathrm{m} / \mathrm{z}$ ratio was recorded from 50 to 600. All HPLC UV and MS data were recorded by Agilent Chemstation. 


\section{Results and discussion}

After keeping catholyte A and B in an Ar-filled glove-box for at least one week, 450 ul of each catholyte was mixed and reacted with a mixture of 500ul DME and 50ul methyl triflate. The mixtures after derivatization was sealed in vials and analyzed by HPLC/UV and MS immediately. The chromatograms are shown in figure 1A, where it's clearly evident that catholyte B had more polysulfide species with longer sulfur chains than those in catholyte A. This observation was expected since more elemental sulfur was in catholyte B than in catholyte A. More elemental sulfur resulted in the higher concentration of polysulfide species with longer sulfur chains [32]. The cyclic voltammograms of the catholytes are shown in figure $1 \mathrm{~B}$. The reduction peak at around $2.0 \mathrm{~V}\left(\mathrm{vs} \mathrm{Li} / \mathrm{Li}^{+}\right)$, which was believed to be the reduction of long chain polysulfides [7], is only evident for catholyte B. The cyclic voltammetry is consistent with that of chromatograms.

It is worth noting how the chromatographic peaks were assigned to each derivatized polysulfide species in figure 1A. To ensure the results with high confidence, the assignment of the polysulfide species is based on three methods: comparison with the standard of derivatized polysulfides, identification through mass spectrometry $(\mathrm{m} / \mathrm{z})$, and correlation with retention property of derivatized polysulfide species. As shown in the supporting information (figure S-1, and table S-1), in this study all assignments of derivatized species $\left(\mathrm{Me}_{2} \mathrm{~S}_{\mathrm{n}}, \mathrm{n}=3\right.$ to 7) were performed with at least two of the methods mentioned above, with the exception of $\mathrm{Me}_{2} \mathrm{~S}_{8}$, which was solely based on the linear relationship between retention time and sulfur chain, and $\mathrm{Me}_{2} \mathrm{~S}_{2}$, which was only based on the commercial standard. The minor chromatographic peaks after 12 min were assumed to be methylated polysulfide species with more than 8 sulfur atoms [27].

\section{[Figure 1]}


It has been demonstrated that the polysulfides in the electrolyte of the discharged Li-S battery can be quantitatively and qualitatively determined by ex-situ derivatization tandem with either HPLC-UV [30] or HPLC-MS [31]. However the application of ex-situ derivatization methods on the study of the electrochemical reduction mechanism of sulfur has its limitation due to the fast kinetics of the subsequent complicated disproportionation and chemical reactions of polysulfides in the electrolyte. The electrochemically generated polysulfide ions could become disproportionated during discharge and during the sampling process of ex-situ derivatization, which can last from minutes to hours. The ex-situ derivatization method may not be able to differentiate the polysulfide ions formed electrochemically from those formed from the subsequent chemical processes. Therefore, the proposed mechanism based on ex-situ analytical methods may not be accurate [33].

To alleviate the influence of the subsequent rapid chemical reactions of the electrochemically produced polysulfide species, an in-situ derivatization method was used in this study. The derivatization reagent, methyl triflate, was mixed into the catholyte. Therefore, the polysulfides were derivatized as soon as they formed on the electrode, before having a chance to disproportionate or become reacted. It should be noted that the derivatization between polysulfides and methyl triflate has been reported as a single phase, fast reaction [26]. The kinetic and isotopic mixing experiments indicated that the derivatization reactions are faster than the potential disproportionation reactions and the chemical reactions between the polysulfide ions themselves and with elemental sulfur.

A series of CV experiments were carried out and the results are shown in figure 2. Curve 1 in figure 2 shows the cyclic voltammogram of $20 \mathrm{mM}$ methyl triflate in LiTFSi/DME solution. No redox peaks can be observed in curve 1. Methyl triflate is electrochemically stable within the potential range. Curves 2 and 3 show cyclic voltammograms of catholyte A after derivatization with methyl triflate and catholyte A alone, respectively. The redox peaks 
in curve 3 demonstrate that the polysulfide ions in catholyte A before derivatization can either be reduced or oxidized. After all the polysulfide ions in catholyte A have been derivatized by methyl triflate, there are no signs of redox reactions for the derivatized catholyte A (curve 2). The disappearance of redox peaks of the polysulfide ions reveals that the derivatized polysulfide ions are inert to electrochemical redox reactions. Taking into consideration the fast kinetics for the derivatization reaction, the in-situ derivatization during electrochemical measurements paved a new avenue for the investigation of the sulfur redox reaction mechanism. Any polysulfides produced at a certain potential or depth of discharge can be captured by methyl triflate and the derivatized polysulfide ions are immune to both chemical and electrochemical reactions.

\section{[Figure 2]}

Curves 4 and 5 in figure 2 show the cyclic voltammograms of a sulfur saturated LiTFSi/DME solution without and with the addition of $20 \mathrm{mM}$ of methyl triflate, respectively. Three major differences can be observed between curves 4 and 5. With the addition of methyl triflate, the two-reduction peak profile in curve 4 became a one-reduction peak profile; no oxidation peak can be observed in curve 5 and there is a negative shift of about $100 \mathrm{mV}$ of the sulfur reduction potential in curve 5. It can be confidently stated that all the polysulfide ions generated during the reduction of sulfur were derivatized, due to the fact that there was no

oxidation current in curve 5 . The negative shift of the reduction peak in curve 5 was due to the change of redox couple from $S / S_{x}{ }^{2-}$ to $S / R^{x} R^{2-}$. It is shown in figure $S-5$, unless all the polysulfides become derivatized, the addition of methyl triflate did not change the reduction potential of elemental sulfur. It needs to be pointed out that there was no chemical interaction between sulfur and methyl triflate. As shown in figure S-2 in the supplemental material, the chromatograms of the sulfur saturated catholytes with and without the addition of methyl triflate are identical. It is also worth to emphasizing that the one-electron transfer intermediate, 
$\mathrm{S}_{8}^{-{ }^{-}}$radical ion, cannot be captured by the in-situ derivatization, because $\mathrm{S}_{8}{ }^{-}{ }^{-}$is not a strong nucleophile as other dianion $\mathrm{S}_{\mathrm{n}}{ }^{2-}$. Therefore, the in-situ derivatization captured the very first dianions formed electrochemically, whether it is through 2-e reduction of sulfur or 1-e reduction of $\mathrm{S}_{8}{ }^{-}$.

Figure 3 shows the chromatograms of the electrolytes from Li-S batteries polarized at 2.3 $\mathrm{V}$ and derivatized by the in-situ and ex-situ methods. Interestingly, $\mathrm{Me}_{2} \mathrm{~S}_{8}$ was hardly present in the catholyte in which methyl triflate was added, and neither were $\mathrm{Me}_{2} \mathrm{~S}_{7}$ nor $\mathrm{Me}_{2} \mathrm{~S}_{6}$ the most abundant species in that catholyte either. In other words, the immediate product of the electrochemical reduction of sulfur was not $\mathrm{S}_{8}{ }^{2-}$. Schemes 1 to 3 provide overviews of the reported mechanisms for the first reduction wave of the reduction of sulfur, where $\mathrm{S}_{8}{ }^{2-}$ was believed to be the major initial product of the sulfur reduction. The direct experimental evidence in figure 3 clearly shows otherwise. However, significantly more long chain polysulfide ions were found in the ex-situ derivatized catholyte. Even though both Li-S cells were discharged to similar capacity, more $\mathrm{S}_{8}$ was consumed in the catholyte without the addition of methyl triflate. Table 1 tabulates the comparison of the actual remaining sulfur (based on the method reported in reference [34]) in the catholyte after discharge with that of the theoretical amount (the initial amount of sulfur minus the amount consumed electrochemically). Obviously, the electrochemical reduction cannot account for all the sulfur consumption in the ex-situ derivatization. The additional consumption of sulfur can be attributed to the chemical reactions between the polysulfide and elemental sulfur. When methyl triflate was added to the catholyte, the polysulfide ions were immediately derivatized and subsequent chemical reactions with sulfur were avoided. The slightly higher sulfur content could be the result of a side electrochemical reaction (e.g. forming surface layer), since we assumed that all of the charges were used to reduce sulfur. This observation is important because it revealed that first, the chemical reaction between the polysulfides and 
sulfur had fast kinetics and may reach a chemical equilibrium quickly; second, traditional exsitu analysis methods may not be suitable for the investigation of the sulfur reaction mechanism. For example, the soluble polysulfides were derivatized through benzylation and analyzed by LC-MS and NMR [33]. Since the analysis was done ex-situ and the derivatization took hours to complete, additional interactions between the electrochemically formed polysulfides with either elemental sulfur or with themselves likely took place. Therefore, the polysulfides detected ex-situ may not be those formed electrochemically. In fact, the polysulfides detected in the $2.1 \mathrm{~V}$ region (closed to $2.3 \mathrm{~V}$ vs. $\mathrm{Li}$ ) were consistent with the polysulfides found in our ex-situ experiments. Since ex-situ analysis was used in most of the mechanistic studies for Li-S batteries, it is not surprising that the long chain polysulfides (e.g. $\mathrm{S}_{8}{ }^{2-}$ and $\mathrm{S}_{7}{ }^{2-}$ ) were reported as the initial reaction products.

\section{[Figure 3]}

\section{[Table 1]}

It is worth to emphasizing that both elemental sulfur and polysulfide ions were dissolved in the electrolyte, no solid sulfur electrode was used. In the catholyte C, the concentration of the saturated sulfur solution was about $5 \mathrm{mM}$ [34]. Excess amount (20 mM) of methyl triflate was added to ensure that the electrochemcial formed polysulfides would be derivatized immidiately and provented from any subsequent chemical or electrochemcial reactions. The total amount of sulfur in both in-situ and ex-situ experiments were the same. The very polysulfide formed in the electrochemcial reductin of elemental sulfur was identified in the in-situ experiment, while the distribution of the polysulfide ions at semi-eqilibrium was determined in the ex-situ experiment.

Based on the evidence from the in-situ analysis, $\mathrm{S}_{8}{ }^{2-}$ was not the major product of the initial electrochemical reduction of sulfur; instead multiple polysulfide ions with shorter chain 
lengths were detected. The observation of high concentrations of $\mathrm{S}_{8}{ }^{2-}$ could be produced by subsequent chemical reactions between short chain polysulfides and elemental sulfur. Instead of the multiple sequential steps pathway mechanisms shown in schemes 1-3, the first step of the reduction of sulfur may involve multiple parallel pathways and -S-S- bond cleavage along with electron transfer. It should be noted that the derivatization did not alter the chemical structure of the polysulfide ions e.g. the chain length. In the supplemental material, the same polysulfide solution was derivatized with different reagents and analyzed with different solvents (mobile phase), the results shown in figure S-4 (in supporting information) are identical. The in-situ derivatization paved a new avenue for the investigation of the mechanism of sulfur electrochemical reduction, but more careful investigations are still needed to better understand details of that mechanism.

\section{Conclusion}

Unlike ex-situ analysis, in which polysulfide ions were given adequate time to react with elemental sulfur, in-situ and rapid derivatization was reported to capture the true products formed during electrochemical reduction of sulfur. It was found that the major polysulfide species formed at the first reduction wave of elemental sulfur are the $\mathrm{S}_{4}{ }^{2-}$ and $\mathrm{S}_{5}{ }^{2-}$ species instead of the widely accepted $\mathrm{S}_{8}{ }^{2-}$ and $\mathrm{S}_{6}{ }^{2-}$ species, which were found to be the result of subsequent chemical reactions with elemental sulfur.

\section{Acknowledgements}

The authors from UWM and BNL are indebted to the Assistant Secretary for Energy Efficiency and Renewable Energy, Office of Vehicle Technologies, under the program of Vehicle Technology Program, under Contract Number DE-SC0012704. The authors from WUT are grateful for the supports from Fundamental Research Funds for the Central Universities (WUT: 2015-IB-001). 


\section{References}

[1] P.G. Bruce, S.A. Freunberger, L.J. Hardwick, J.-M. Tarascon, Nat. Mater. 11 (2012) $19-29$.

[2] J. Badoz-Lambling, R. Bonnaterre, G. Cauquis, M. Delamar, Georgette Demange, Electrochim. Acta 21 (1976) 119-131.

[3] J. Paris, V. Plichon, Electrochim. Acta 26 (1981) 1823-1829.

[4] H. Yamin, A. Gorenshtein, J. Penciner, Y. Sternberg, E. Peled, J. Electrochem. Soc. 135 (1988) 1045-1048.

[5] B.S. Kim, S.M. Park, J. Electrochem. Soc. 140 (1993) 115-122.

[6] A. Evans, M. I. Montenegro, D. Pletcher, Electrochem. Commun. 3 (2001) 514-518.

[7] P. Leghie, J.-P. Lelieur, E. Levillain, Electrochem. Commun. 4 (2002) 406-411.

[8] D.-H. Han, B.-S. Kim, S.-J. Choi, Y. Jung, J. Kwak, S.-M. Park, J. Electrochem. Soc. 151 (2004) E283-E290.

[9] N.S.A. Manan, L. Aldous, Y. Alias, P. Murray, L.J. Yellowlees, M.C. Lagunas, C. Hardacre, J. Phys. Chem. B 115 (2011) 13873-13879.

[10] J.-T. Yeon, J.-Y. Jang, J.-G. Han, J. Cho, K. T. lee, N.-S. Choi, J. Electrochem. Soc. 159 (2012) A1308-A1314.

[11] M. Hagen, P. Schiffels, M. Hammer, S. Dorfler, J. Tubke, M.J. Hoffmann, H. Althues, S. Kaskel, J. Electrochem. Soc. 160 (2013) A1205-A1214.

[12] M.U.M. Patel, R. Dominko, ChemSusChem 7 (2014) 2167-2175. 
[13] A.S. Baranski, W. R. Fawcett, C.M. Gilbert, Anal. Chem. 57 (1985) 166-170.

[14] H. Yamin, J. Penciner, A. Gorenshtan, M. Elam, E. Peled, J. Power Sources 14 (1985) 129-134.

[15] Y. Jung, S. Kim, B.-S. Kim, D.-H. Han, S.-M. Park, J. Kwak, Int. J. Electrochem. Sci. 3 (2008) 566-577.

[16] F. Gaillard, E. Levillain, J. Electroanal. Chem. 398 (1995) 77-87.

[17] E. Levillain, F. Gaillard, P. Leghie, A. Demortier, J.P. Lelieur, J. Electroanal. Chem. 420 (1997) 167-177.

[18] H.L. Wu, L. A. Huff, A.A. Gewirth, ACS Appl. Mater. \& Interfaces 7 (2015) 17091719.

[19] M. Vijayakumar, N. Govind, E. Walter, S.D. Burton, A. Shukla, A. Devaraj, J. Xiao, J. Liu, C.M. Wang, A. Karim, S. Thevuthasan, Phys. Chem. Chem. Phys. 16 (2014) 1092310932.

[20] H. S. Ryu, H. J. Ahn, K. W. Kim, J. H. Ahn, J. Y. Lee, J. Power Sources 153 (2006) 360-364.

[21] H. S. Ryu, Z. P. Guo, H. J. Ahn, G. B. Cho, H.K. Liu, J. Power Sources 189 (2009) 1179-1183.

[22] M.A. Lowe, J. Gao, H.D. Abruna, R. Soc. Chem. Adv. 4 (2014) 18347-18353.

[23] L.J. Wang, T. Zhang, S.Q. Yang, F.Y. Cheng, J. Liang, J. Chen, J. Energy Chem. 22 (2013) 72-77.

[24] B. Wang, S. M. Alhassan, S.T. Pantelides, Phys. Rev. Appl. 2 (2014) 034004. 
[25] R.S. Assary, L. A. Curtiss, J.S. Moore, J. Phys. Chem. C 118 (2014) 11545-11558.

[26] A. Kamyshny, A. Goifman, J. Gun, D. Rizkov, O. Lev, Environ. Sci. \& Technol. 38 (2004) 6633-664.

[27] D. Rizkov, O. Lev, J. Gun, B. Anisimov, I. Kuselman, Accredit. Qual. Assur. 9 (2004) 399-403.

[28] A. Kamyshny, I. Ekeltchik, J. Gun, O. Lev, Anal. Chem. 78 (2006) 2631-2639.

[29] A. Kamyshny, J. Gun, D. Rizkov, T. Voitsekovski, O. Lev, Environ. Sci. \& Technol. 41 (2007) 2395-2400.

[30] C. Barchasz, F. Molton, C. Duboc, J.-C. Lepretre, S. Patoux, F. Alloin, Anal. Chem. 84 (2012) 3973-3980.

[31] D. Zheng, D. Qu, X.-Q. Yang, X. Yu, H.-S. Lee, D.Y. Qu, Adv. Energy Mater. (2015) DOI:19.1002/aenm.201401888.

[32] R.D. Rauh, F.S. Shuker, J.M. Marston, S.B. Brummer, J. Inorg. Nucl. Chem. 39 (1977) 1761-1766.

[33] A. Kawase, S. Shirai, Y. Yanoto, R. Arakawa, T. Takata, Phys. Chem. Chem. Phys. 16 (2014) 9344-9350.

[34] D. Zheng, X. Zhang, C. Li, M.E. Mckinnon, R.G. Sadok, D. Qu, X. Yu, H.-S. Lee, X.Q. Yang, D.Y. Qu, J. Electrochem. Soc. 162 (2015) A203-A206. 
Scheme 1:

$\mathrm{S}_{8 \mathrm{C}} \stackrel{2 \mathrm{e}^{-}}{\longrightarrow} \mathrm{S}_{8 \mathrm{~L}}^{2-} \stackrel{\text { Disproportionation }}{\longrightarrow} \mathrm{S}_{6 \mathrm{~L}}^{2-}+1 / 4 \mathrm{~S}_{8 \mathrm{C}}$

Scheme 2:

$\mathrm{S}_{8 \mathrm{C}} \stackrel{\mathrm{e}^{-}}{\longrightarrow} \mathrm{S}_{8 \mathrm{C}}^{--} \longrightarrow \mathrm{S}_{8 \mathrm{~L}}^{\cdot-} \stackrel{\mathrm{e}^{-}}{\longrightarrow} \mathrm{S}_{8 \mathrm{~L}}^{2-} \stackrel{\text { Disproportionation }}{\longrightarrow} \mathrm{S}_{6 \mathrm{~L}}^{2-}+1 / 4 \mathrm{~S}_{8 \mathrm{C}}$

Scheme 3:

$\mathrm{S}_{8 \mathrm{C}} \stackrel{\mathrm{e}^{-}}{\longrightarrow} \mathrm{S}_{8 \mathrm{C}}^{--} \stackrel{\mathrm{e}^{-}}{\longrightarrow} \mathrm{S}_{8 \mathrm{~L}}^{2-} \stackrel{\text { Disproportionation }}{\longrightarrow} \mathrm{S}_{6 \mathrm{~L}}^{2-}+1 / 4 \mathrm{~S}_{8 \mathrm{C}}$ 
Figure 1
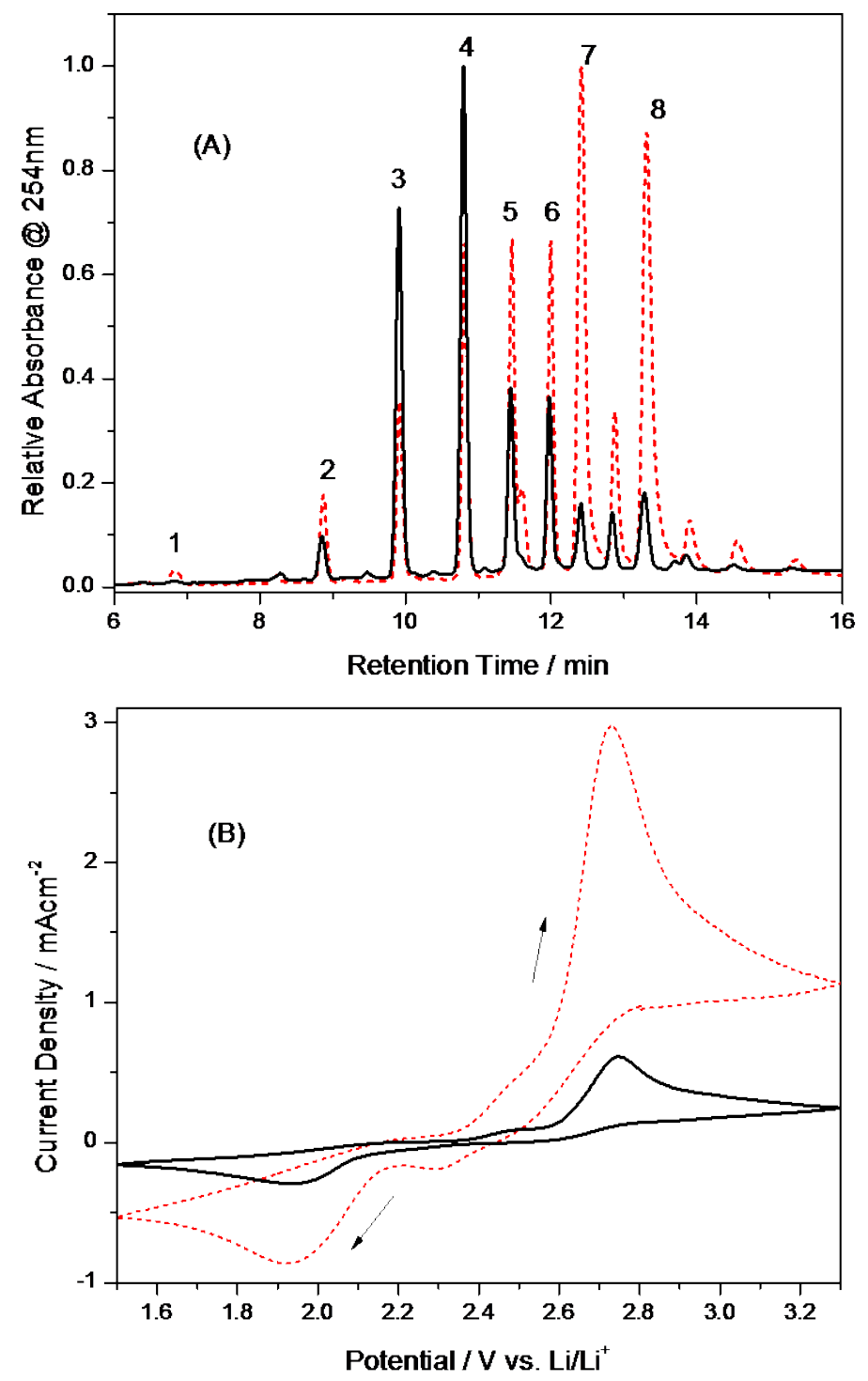
Figure 2

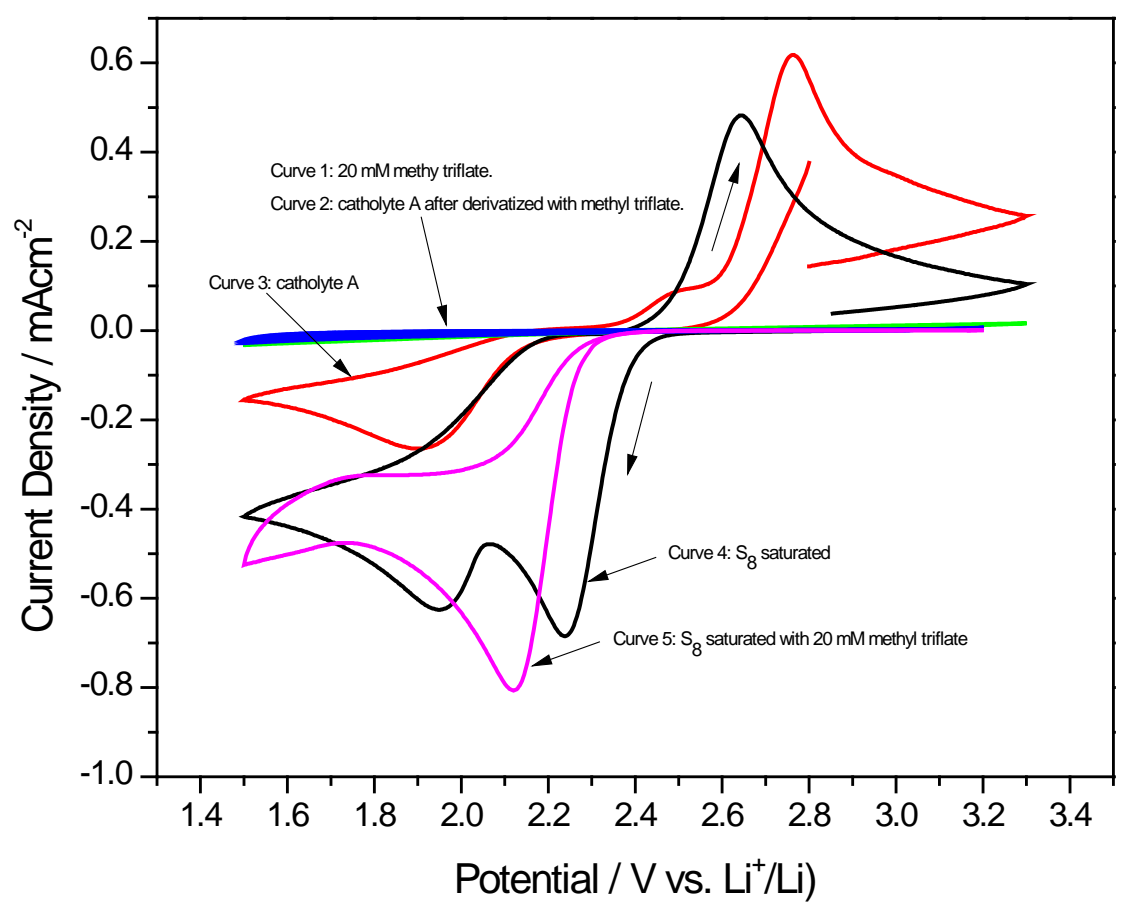


Figure 3

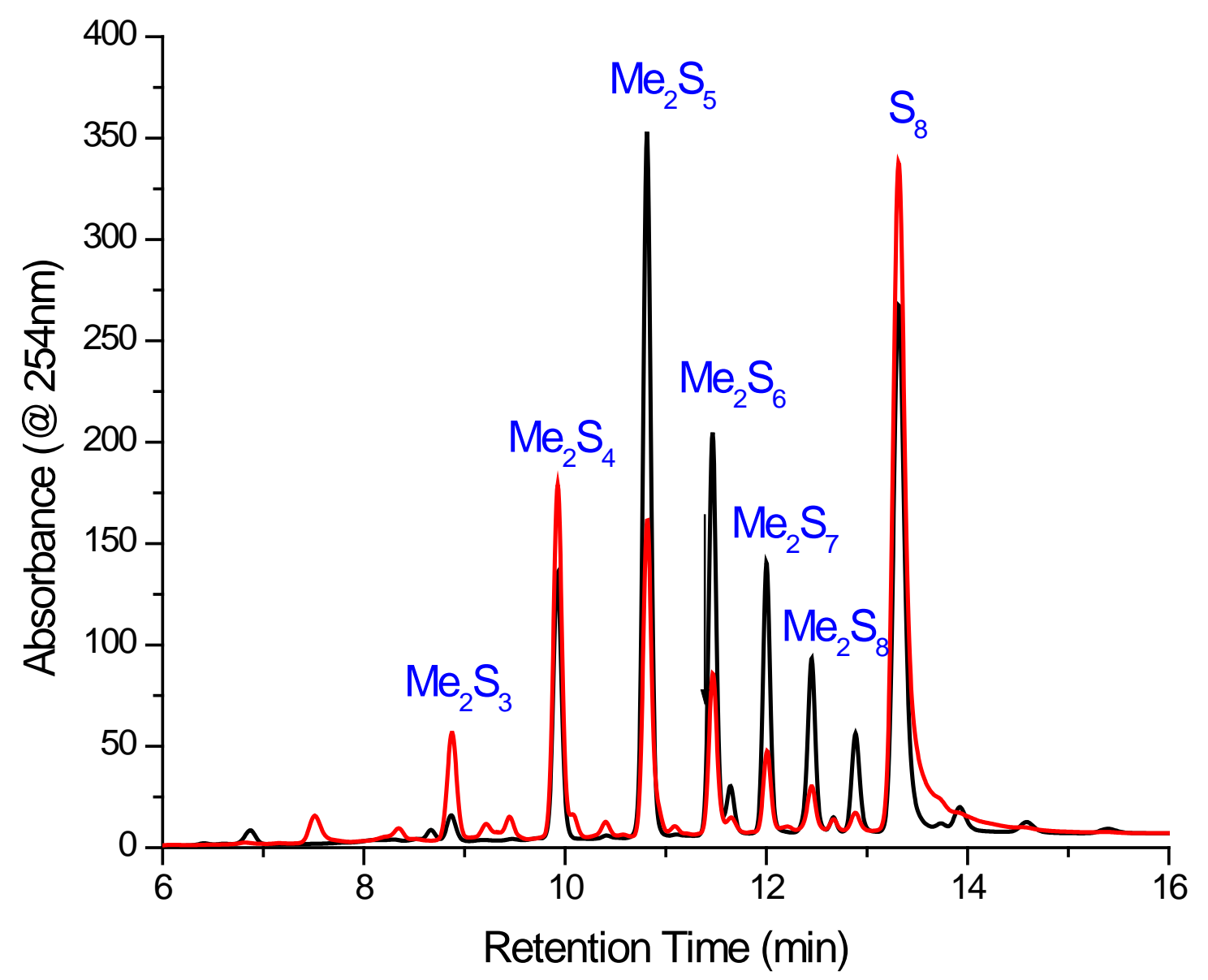


Table 1

\begin{tabular}{|l|l|l|l|}
\hline $\begin{array}{l}\text { Derivatization } \\
\text { method }\end{array}$ & $\begin{array}{l}\text { Discharge capacity } \\
\text { from polarization at } \\
2.3 \mathrm{~V}(\mathrm{mAh})\end{array}$ & $\begin{array}{l}\text { Percentage of elemental sulfur } \\
\text { left from theoretical } \\
\text { calculation based on 2- } \\
\text { electron transfer }\end{array}$ & $\begin{array}{l}\text { Percentage of elemental } \\
\text { sulfur left from HPLC/UV } \\
\text { measurement }\end{array}$ \\
\hline In-Situ & 1.42 & $34.0 \%$ & $37.9 \%$ \\
\hline Ex-Situ & 1.43 & $33.3 \%$ & $24.9 \%$ \\
\hline
\end{tabular}

